\title{
Caracterização da Clientela das Clínicas-Escola de Cursos de Psicologia da Região Metropolitana de Porto Alegre
}

\author{
Client's Profile of University's Psychology Clinics \\ from Porto Alegre's Metropolitan Region
}

\author{
Paula von Mengden Campezatto* \& Maria Lúcia Tiellet Nunes \\ Pontifícia Universidade Católica do Rio Grande do Sul, Porto Alegre, Brasil
}

\begin{abstract}
Resumo
São diversos os estudos sobre a caracterização da clientela de clínicas-escola de Psicologia no Brasil. Todavia, há escassez de pesquisas desta natureza no Rio Grande do Sul. Este trabalho se propõe a realizar uma pesquisa de levantamento das características sociodemográficas e clínicas da população que buscou atendimento em 2004 nas dez clínicas-escola da Região Metropolitana de Porto Alegre. É possível reconhecer a similaridade dos achados com a grande maioria dos estudos, permitindo-se a inferência de um perfil típico do paciente que busca atendimento em clínicas-escola no Brasil: crianças, do sexo masculino, encaminhadas por escolas devido a dificuldades de aprendizagem ou comportamento e mulheres jovens, que buscam atendimento espontaneamente devido a conflitos relativos ao comportamento afetivo. Também se destaca a dificuldade em pesquisar sobre o tema em função da ausência de registros padronizados, adequados e bem preenchidos pelas clínicas-escola. Palavras-chave: Atendimento psicológico; clínica-escola; clientela; características sociodemográficas; características clínicas.
\end{abstract}

\begin{abstract}
Although many studies about Psychology School-Clinics have been conducted in Brazil, there are few in Rio Grande do Sul. So that, this paper is a survey's research about sociodemographic and clinical characteristics of people who reached out for psychological service in 2004 at 10 Psychology School-Clinics in metropolitan area of Por to Alegre. The results showed similarity between most of other studies that had been previously conducted in Brazil and the present one, allowing the inference of two typical profiles in Psychology SchoolClinics in Brazil: children, male, referred by schools because of learning or behavior problems and young women, who sought, on their own initiative, psychological consultation due to affective problems. It is also important to mention how difficult it is to research about this topic, because there's no standardized, adequate and well-filled out inpatient registration in Psychology School-Clinics.

Keywords: Psychological service; psychology school-clinic; client profile; sociodemographic characteristics, clinical characteristics.
\end{abstract}

Clínicas-escola de Psicologia (C-e) são serviços obrigatórios pela legislação que dispõe sobre os Cursos de Formação em Psicologia no Brasil e regulamenta a profissão de psicólogo em nosso país (Lei nº 4.119, 1962). Essas instituições possuem uma dupla-função, conforme a ampla literatura da área, dentre eles (Ferreira, 1998; Güntert et al, 2000; Terzis \& Carvalho, 1988): proporcionam ao estagiário o exercício supervisionado da prática clínica, ao mesmo tempo que permitem à Universidade cumprir um de seus papéis sociais: prestação de serviços à comunidade Apesar de configurarem umas das poucas instituições de prestação de serviços psicológicos acessíveis fi-

\footnotetext{
* Endereço para correspondência:

Pontifícia Universidade Católica do Rio Grande do Sul, Av Ipiranga, 6681. Prédio 11, $9^{\circ}$ andar, sala 928, Porto Alegre, RS,90619-900.E-mails: paulavmc@hotmail.com ou tiellet@pucrs.br

A pesquisa que deu origem a esse artigo foi financiada pelo CNPq e é parte de uma Disser tação de Mestrado em Psicologia Clínica.
}

nanceiramente a uma grande parcela da população brasileira (Barbosa \& Silvares, 1994), as C-e foram constituídas a partir de uma obrigatoriedade legal e organizadas de acordo com as necessidades e possibilidades dos encarregados da educação e formação do futuro psicólogo, e não, necessariamente, segundo as reais necessidades da população a ser atendida (Barbosa \& Silvares, 1994; Calejon, 1995).

De acordo com a pesquisa realizada por Ancona-Lopez (1983b), as C-e funcionam de forma bastante contraditória, na medida em que se dispõem a oferecer serviços psicológicos; mas a maioria da clientela é encaminhada ou permanece em longas listas de espera por atendimento. Em sua pesquisa, constatou que $54,1 \%$ da clientela abandonou os atendimentos, e apenas 4,6\% cumpriram os objetivos propostos, com alta. As C-e, muitas vezes, parecem desconhecer as reais necessidades da clientela que as procura, correndo o risco de não a atenderem adequadamente. Assim, V. L. A. Peres (1997) considera que essas devem se perguntar quem são, de onde vêm, o que buscam, como 
vivem e quais são as expectativas dos seus clientes. Um passo inicial para tal investigação é o levantamento das características sociodemográficas da população que procura seus serviços, descrição esta considerada como parte de resultados de diagnósticos institucionais. Tais resultados podem auxiliar a definir intervenções psicológicas mais adequadas (Silvares, 1996).

Somente após esse conhecimento inicial, é que se poderá propor mudanças na estruturação de cada C-e e formular estratégias de ação específicas, a fim de que possam oferecer serviços mais adequados e eficazes para a população que delas se utiliza (Barbosa \& Silvares, 1994; Romaro \& Capitão, 2003; Schmidt, 1992; Silvares, 1996), pois também é objetivo das C-e proporcionar condições que favoreçam a formação de seus estagiários e desenvolver pesquisas para auxiliar na qualidade da formação destes, refletindo na qualidade dos atendimentos oferecidos (Arcaro \& Mejias, 1990; Calejon, 1995; Guerrelhas \& Silvares, 2000).

São diversos os estudos sobre este tema no Brasil. Porém, percebe-se que estes são, em sua grande maioria, oriundos do estado de São Paulo, e muito poucos das outras regiões. Silvares (1996) e Levandowski (1998) realizaram revisões acerca das produções desta natureza, constatando que a maioria das universidades brasileiras não tem desenvolvido estudos sobre este tema. Há escassez deste tipo de estudo no Rio Grande do Sul, estado que concentra 23 Cursos de Psicologia.

Portanto, o objetivo desta pesquisa é conhecer a população que buscou atendimento psicológico em 2004 nas clínicas-escola da Região Metropolitana de Porto Alegre, abrangendo faixa etária, escolaridade, ocupação, nível socioeconômico, motivo da busca de atendimento, por quem são encaminhados e o desfecho de seu atendimento nas Ce. As questões serão aplicadas a toda a população atendida, incluindo crianças, ainda que algumas questões (ocupação, estado civil) não se apliquem diretamente a esta faixa etária.

\section{Método}

\section{Delineamento}

Este trabalho se constituiu em uma pesquisa quantitativa, descritiva e de levantamento. Partindo desses pressupostos, a escolha metodológica da pesquisa se deu a partir de fatores que beneficiassem a coleta dos dados, ou seja, a possibilidade de atingir um maior número de instituições; menores gastos; rapidez na obtenção dos resultados; e garantia do anonimato dos sujeitos - permitindo, com isso, que as pessoas respondessem ao questionário no momento em que julgassem conveniente (Goodwin, 1995).

\section{Participantes}

A fonte de informações é oriunda de todas as C-e vinculadas aos cursos de Psicologia, distribuídas na Região Metropolitana de Porto Alegre, representadas por seus coordenadores. Nesta região, situam-se 10 das 23 clínicas-escola de Psicologia existentes no Estado do Rio Gran- de do Sul e compreende os municípios de Porto Alegre, Canoas, Gravataí, Guaíba, Novo Hamburgo, São Jerônimo, São Leopoldo, Taquara e Torres (Instituto Brasileiro de Geografia e Estatística [IBGE], 2004), com as quais foram realizados contatos prévios para apresentar os objetivos da pesquisa à direção cientifica e/ou de ensino do local.

\section{Instrumento}

A elaboração do instrumento da pesquisa se deu por meio de um levantamento amplo e aprofundado da literatura pertinente, juntamente com a experiência de membros do Grupo de Pesquisa 'Avaliação e Intervenção em Psicoterapia e Psicossomática' da Pontifícia Universidade Católica do Rio Grande do Sul (PUCRS) no tema relativo às C-e. Foi elaborado um questionário de forma a contemplar as informações necessárias para atingir os objetivos deste estudo, dispostas em questões objetivas e abertas, a serem preenchidas de acordo com o funcionamento de cada C-e e com dados registrados em Fichas de Atendimento/ Prontuários/Livros de Registro. Para tanto, o instrumento criado por Ghazzi (2000) serviu como base, pois trata, também, de um questionário elaborado para levantar dados a respeito de características do trabalho de Estimulação Precoce no Rio Grande do Sul.

Em uma primeira parte do questionário, são verificadas as características dos atendimentos prestados pelas C-e (localização da C-e, locais onde as atividades são exercidas, modalidades terapêuticas, abordagens teóricas, modo como são realizadas atividades de Triagem, uso ou não de Termo de Consentimento Livre e Esclarecido, critérios para receber atendimento na instituição e critérios de alta terapêutica), dados explorados em outro artigo derivado desta Dissertação de Mestrado (Campezatto \& Nunes, 2007). Na segunda parte, as questões são relativas a características sócio-demográficas da população atendida nas C-e (sexo, idade, estado civil, ocupação, escolaridade, renda familiar), bem como as características clínicas da população atendida (número de atendimentos, altas e abandonos). A questão a respeito do motivo da busca de atendimento da clientela se constituiu em 12 agrupamentos de categorias, oriundos de outros estudos semelhantes sobre o tema (Ancona-Lopez, 1983a; Barbosa \& Silvares, 1994; Carvalho \& Telles, 2001; Graminha \& Martins, 1993; Levandowski, 1998; Romaro \& Capitão, 2003). A partir de uma análise de conteúdo (Bardin, 1988) das categorias propostas pelos autores acima citados, foi construída a distribuição de categorias utilizadas nessa pesquisa, por serem entendidas como um grupo de itens suficientemente amplo para permitir às C-e alocarem as informações dos prontuários e, ao mesmo tempo, capaz de orientar essa alocação de informações. Ainda assim, as autoras compreendem que uma transposição desse tipo pode gerar dificuldades para quem preenche o formulário ao buscar a correspondente categoria no questionário da pesquisa, o que é entendido como possível limitação do estudo.

Foi realizado um estudo piloto com professores supervisores de uma das C-e participantes deste estudo, no qual foi constatado que os sujeitos eram capazes de responder 
às questões contidas no questionário que tinham por objetivo conhecer o funcionamento das C-e. Todavia, estes mesmos professores desconheciam dados sociodemográficos da clientela que busca atendimento, relatando que a C-e não possuía estes dados sistematizados, impossibilitando o preenchimento de tais questões.

\section{Procedimentos de Coleta e Análise de Dados}

Para realizar a coleta de dados, o questionário foi entregue dentro de um envelope lacrado, pessoalmente ou via correio, a cada coordenador(a), juntamente com uma carta de apresentação, explicando o porquê da pesquisa, por quem deveria ser respondida e assegurando a não-identificação dos respondentes. Juntamente com os questionários, foram enviados envelopes endereçados às pesquisadoras e selados, para que lhes fossem remetidos de volta.

Os questionários recebidos foram submetidos à análise de dados. Os dados coletados que dizem respeito à caracterização da clientela são relativos a questões objetivas, sendo que as categorias foram elaboradas pelas pesquisadoras a partir de outras pesquisas encontradas na revisão da literatura, como as que serão apresentadas nas Tabelas 7, 8 e 9. As questões objetivas foram analisados a partir de estatística descritiva, através dos programas estatísticos «SPSS for Windows», versão 11.5, e «Microsoft Excel for Windows», abrangendo médias, freqüências e porcentagens. Também foi utilizado o teste do Qui-quadrado, para verificar a associação entre duas variáveis analisadas neste estudo.

\section{Procedimentos Éticos}

Os respondentes desta pesquisa receberam um Termo de Consentimento Livre e Esclarecido, convidando-os a participarem do estudo, o qual foi assinado e devolvido às pesquisadoras. Este estudo foi aprovado pela Comissão Científica da Faculdade de Psicologia da PUCRS e pelo Comitê de Ética em Pesquisa da PUCRS.

\section{Resultados e Discussão}

As C-e convidadas a participarem deste estudo estão listadas na Tabela 1, disposta abaixo, seguindo nomenclatura fictícia, acompanhada pelo número de sujeitos atendidos no ano de 2004. Dentre as dez C-e que foram convidadas a participarem deste estudo, uma delas $(J)$ não respondeu a nenhuma das tentativas de contato das pesquisadoras - telefonemas, correspondência e correio eletrônico, sendo desconsiderada na pesquisa. A C-e I, pertencente a um curso de Psicologia ainda recente, apresentou como justificativa, para não responder à pesquisa, o fato de a Ce ainda estar em tratativas de regulamentação com o Ministério da Educação (MEC), não sendo, assim, local de estágio para os alunos da Graduação. Como não foi possível contar com os resultados das clínicas $I$ e $J$, estas foram excluídas das demais tabelas apresentadas neste estudo. Três C-e $(A, B, G)$ solicitaram auxílio das pesquisadoras na coleta dos dados necessários para responder ao instrumento, bem como para constituir um banco de dados para uso próprio. As demais $(C, D, E, F, H)$ seguiram as instruções propostas, devolvendo o questionário preenchido conforme solicitado na carta de apresentação e nas instruções de preenchimento; o número de sujeitos apresentado pela clínica $D$ merece ressalvas, pois é relativo aos atendimentos realizados apenas no segundo semestre de 2004, devido à impossibilidade de acessar os dados do primeiro semestre daquele ano no momento.

A Tabela 1 permite verificar, em primeiro lugar, uma diversidade em termos da quantidade de pacientes atendidos por cada C-e no período de um ano, demonstrado pelo alto desvio-padrão $(\sigma=420,835)$.

Tabela 1

Número de Pacientes que Buscaram Atendimento nas Clínicas-Escola de Cursos de Psicologia da Região Metropolitana de Porto Alegre

\begin{tabular}{ccl}
\hline Clínicas-escola & N. ${ }^{\circ}$ de pacientes & \multicolumn{1}{c}{$\%$} \\
\hline A & 541 & 16,04 \\
B & 1438 & 42,65 \\
C & 200 & 5,93 \\
D & 45 & 1,33 \\
E & 177 & 5,25 \\
F & 547 & 16,22 \\
G & 299 & 8,87 \\
H & 125 & 3,71 \\
I & - & - \\
J & - & - \\
\hline Total (n) & 3372 & $100 \%$ \\
\hline Média & 421,5 \\
\hline Desvio Padrão & 420,835 \\
\hline
\end{tabular}

O elevado número total de pacientes atendidos nas C-e da Região Metropolitana de Porto Alegre $(N=3372)$ reafirma a importância social destas clínicas, descrita em estudos anteriores, como uma das poucas instituições de prestação de serviços psicológicos acessíveis financeiramente a uma grande parcela da população brasileira (Barbosa \& Silvares, 1994; Nunes, Campezatto, Cruxên \& Savalhia, 2005). Também é importante ressaltar que os atendimentos das C-e $B, F$ e $G$ são realizados tanto por estudantes de graduação em estágio como por profissionais que já concluíram o curso de Psicologia, seja como alunos de Pós-Graduação ou como profissionais contratados pelas universidades. Esse dado também pode refletir em algumas diferenças nos registros sociodemográficos que seguirão.

A própria análise das fontes de informação desta pesquisa (Fichas de Atendimento/Prontuários/Livros de Registro) já demonstra resultados importantes, uma vez que chama a atenção o número de informações faltantes nos registros, as quais se caracterizam por dado missing no banco de dados, que estará presente como 'não consta' em todas as tabelas que se seguem. De posse desse resultado - o grande número de informações faltosas em diversas das variáveis em estudo -, pode-se discutir a necessidade das instituições pesquisadas de revisar os proce- 
dimentos de anotações dos dados dos pacientes. É possível que o próprio material de registro, que passa a ser uma espécie de diário do percurso do paciente na Instituição, deva ser revisto em termos do que deve conter de informações relevantes no que tange ao tratamento de cada paciente.

Goldim (1998) ressalta a necessidade de que terapeutas estejam atentos para registrar marcos importantes da evolução de cada caso, no sentido de ser este seu dever profissional. Poder-se-ia mesmo pensar que não manter os registros caracteriza-se como negligência, «falta de observação dos deveres profissionais» (p. 124), que é um dos três erros profissionais (imperícia, imprudência e negligência). Além disso, registros incompletos também difi- cultam ou até impedem a realização de pesquisas, tão necessárias para dar retorno da atividade clínica aos próprios terapeutas, aos supervisores e à Instituição.

Talvez haja necessidade das C-e orientarem melhor sua equipe para o preenchimento deste material. É provável que seja preciso algum tipo de acompanhamento do preenchimento do material em supervisão ou em alguma outra instância, principalmente no que diz respeito a psicólogos em formação, que poderão vir a nortear sua futura atuação profissional com base na experiência adquirida na C-e.

A Tabela 2 apresenta uma distribuição dos pacientes atendidos nas C-e deste estudo, de acordo com as variáveis sexo e idade.

Tabela 2

Descrição das Variáveis Sexo e Idade da Clientela das Clínicas-Escola de Cursos de Psicologia da Região Metropolitana de Porto Alegre

\begin{tabular}{|c|c|c|c|c|c|c|c|c|c|c|}
\hline Clínica & $\mathrm{A}$ & $\mathrm{B}$ & $\mathrm{C}$ & $\mathrm{D}$ & $\mathrm{E}$ & $\mathrm{F}$ & G & $\mathrm{H}$ & Total & $\%$ \\
\hline \multicolumn{11}{|l|}{ Sexo } \\
\hline Feminino & 326 & 872 & 64 & 12 & 60 & 565 & 222 & 53 & 2174 & 59,73 \\
\hline Masculino & 214 & 566 & 44 & 14 & 34 & 250 & 77 & 72 & 1271 & 34,92 \\
\hline Não consta & 1 & $\mathrm{O}$ & 92 & 19 & 83 & $\mathrm{O}$ & $\mathrm{O}$ & $\mathrm{O}$ & 195 & 5,36 \\
\hline Total & 541 & 1438 & 200 & 45 & 177 & $815^{3}$ & 299 & 125 & 3640 & $100 \%$ \\
\hline \multicolumn{11}{|l|}{ Faixa Etária } \\
\hline Até 5 anos & 38 & 81 & 9 & 1 & 2 & 22 & 4 & 7 & 164 & 4,46 \\
\hline 6 a 10 anos & 114 & 243 & 27 & 11 & 20 & 124 & 33 & 61 & 633 & 17,21 \\
\hline 11 a 15 anos & 87 & 160 & 24 & 4 & 35 & 92 & 14 & 16 & 432 & 11,74 \\
\hline 16 a 20 anos & 51 & 136 & 5 & $\mathrm{O}$ & 6 & 58 & 67 & 6 & 329 & 8,94 \\
\hline 21 a 25 anos & 47 & 147 & 7 & 1 & 4 & 47 & 60 & 7 & 320 & 8,70 \\
\hline 26 a 30 anos & 45 & 138 & 3 & 3 & 7 & 38 & 41 & 5 & 280 & 7,61 \\
\hline 31 a 35 anos & 45 & 101 & 5 & 3 & 6 & 46 & 28 & 7 & 241 & 6,55 \\
\hline 36 a 40 anos & 37 & 100 & 5 & 1 & 5 & 47 & 9 & 10 & 214 & 5,82 \\
\hline 41 a 45 anos & 17 & 106 & 5 & 2 & 3 & 48 & 12 & 1 & 194 & 5,27 \\
\hline 46 a 50 anos & 16 & 80 & 6 & $\mathrm{O}$ & 2 & 43 & 14 & 1 & 162 & 4,40 \\
\hline 51 a 55 anos & 12 & 67 & 6 & $\mathrm{O}$ & 1 & 53 & 11 & 2 & 152 & 4,13 \\
\hline 56 a 60 anos & 8 & 35 & 3 & $\mathrm{O}$ & $\mathrm{O}$ & 58 & 1 & $\mathrm{O}$ & 105 & 2,85 \\
\hline 61 anos em diante & 17 & 31 & 3 & $\mathrm{O}$ & 3 & 139 & 5 & 1 & 199 & $5,4+1$ \\
\hline Famílias & $\mathrm{O}$ & $\mathrm{O}$ & $\mathrm{O}$ & $\mathrm{O}$ & $\mathrm{O}$ & 39 & $\mathrm{O}$ & $\mathrm{O}$ & 39 & 1,06 \\
\hline Não consta & 7 & 13 & 92 & 19 & 83 & $\mathrm{O}$ & $\mathrm{O}$ & 1 & 215 & 5,84 \\
\hline Total & 541 & 1438 & 200 & 45 & 177 & $854^{1}$ & 299 & 125 & 3679 & $100 \%$ \\
\hline
\end{tabular}

${ }^{1}$ Nesta tabela, o número de pacientes atendidos na C-e $F$ é maior do que o apresentado nas demais, pois foram computados dados relativos aos atendimentos realizados pelos Serviços de Enfermagem e Nutrição da C-e, não tendo sido possível separá-los dos atendimentos psicológicos, foco desta pesquisa.
No que diz respeito ao sexo dos sujeitos, percebe-se que a maioria dos atendimentos é destinada a pacientes do sexo feminino, com 59,73\% (2174 sujeitos), em comparação com os $34,92 \%$ (1271 sujeitos) referentes ao sexo masculino. Quando consideramos a faixa etária, observamos uma elevada concentração de crianças de 6 a 10 anos de idade, que compreende $17,21 \%$ (633 sujeitos) do total da clientela atendida, seguido pela população de adolescentes e jovens 
de 11 a 15 anos (com 11,74\%, 432 sujeitos) e 16 a 20 anos (com 8,94\%, 329 sujeitos), respectivamente.

É comum que crianças enfrentem problemas diversos nos primeiros anos escolares, por fatores de ordem interna e externa, capazes de interferirem na aprendizagem e

Tabela 3

Distribuição da Clientela Total das Clínicas-Escola de Cursos de Psicologia da Região Metropolitana de Porto Alegre por Sexo e Faixa Etária

\begin{tabular}{|c|c|c|c|c|c|c|c|c|}
\hline \multirow{2}{*}{$\begin{array}{l}\text { Sexo } \\
\text { Idade }\end{array}$} & \multicolumn{2}{|c|}{ Feminino } & \multicolumn{2}{|c|}{ Masculino } & \multicolumn{2}{|c|}{ Não Consta } & \multicolumn{2}{|c|}{ Total } \\
\hline & N. ${ }^{\circ}$ & $\%$ & N. ${ }^{\circ}$ & $\%$ & N. ${ }^{\circ}$ & $\%$ & N. ${ }^{\circ}$ & $\%$ \\
\hline Até 10 anos & 311 & 8,54 & 4921 & 3,52 & 1 & 0,03 & 317 & 22,09 \\
\hline 11 a 20 anos & 417 & 11,46 & 344 & 9,45 & $\mathrm{O}$ & $\mathrm{O}$ & 909 & 20,91 \\
\hline 21 a 30 anos & 433 & 11,90 & 167 & 4,59 & $\mathrm{O}$ & $\mathrm{O}$ & 777 & 16,48 \\
\hline 31 a 40 anos & 363 & 9,97 & 92 & 2,53 & $\mathrm{O}$ & $\mathrm{O}$ & 530 & 12,50 \\
\hline 41 a 50 anos & 273 & 7,50 & 81 & 2,23 & $\mathrm{O}$ & $\mathrm{O}$ & 365 & 9,73 \\
\hline 51 a 60 anos & 206 & 5,66 & 51 & 1,40 & $\mathrm{O}$ & $\mathrm{O}$ & 287 & 7,06 \\
\hline 61 anos em diante & 160 & 4,40 & 39 & 1,07 & $\mathrm{O}$ & $\mathrm{O}$ & 211 & 5,47 \\
\hline Não consta & 9 & 0,25 & 5 & 0,14 & 196 & 5,38 & 244 & 5,77 \\
\hline Total & 2172 & $59,67 \%$ & 1271 & $34,92 \%$ & 197 & $5,41 \%$ & 3640 & $100 \%$ \\
\hline
\end{tabular}

A análise estatística da Tabela 3, através do teste Quiquadrado, demonstra que há um nível de significância altamente significativo $(p<0,001)$ ao correlacionar as variáveis sexo e faixa etária. É possível verificar uma concentração de crianças de até 10 anos do sexo masculino (13,52\%; 492 sujeitos). Na faixa etária de 11 a 20 anos, tanto adolescentes do sexo feminino como do masculino buscam atendimento $(11,46 \%$, relativos a 417 sujeitos e $9,45 \%$, relativos a 344 sujeitos, respectivamente). Já entre adultos, a partir de 21 anos de idade, há maior procura de atendimento por pessoas do sexo feminino, sendo os pacientes do sexo masculino muito menos freqüentes.

Outras pesquisas que descrevem a caracterização da clientela de C-e constatam a existência de dois perfis característicos, que estão de acordo com os dados descritos acima. O primeiro perfil, de maior freqüência, é caracterizado, sobretudo, por crianças em idade escolar e por adoles- se constituírem em obstáculos à integração de funções como pensar, sentir, falar e agir. Essas dificuldades tendem a aparecer como queixas de dificuldades de aprendizagem, de acordo com Capellini, Tonelotto e Ciasca (2004).

Ao cruzar as variáveis sexo e faixa etária, obtém-se a Tabela 3, disposta abaixo:

Tabela 4

centes, com concentração na faixa de 6 a 15 anos, predominantemente do sexo masculino, encaminhados por professores, tendo como motivo o insucesso escolar (AnconaLopez, 1983b; Arcaro, 1991; Bernardes da Rosa, Garcia, Domingos \& Silvares, 2000; Boarini \& Borges, 1998; Graminha \& Martins, 1993; Louzada, 2003; Romaro \& Capitão, 2003; Silvares, 1996, 2000; Terzis \& Carvalho, 1988; Wolf, 1988; Yehia, 1994). Capellini, Tonelotto e Ciasca (2004) lembram que as dificuldades do aprender estão presentes em uma incidência de três para um, comparando-se o sexo masculino com o feminino.

O segundo perfil pode ser caracterizado por mulheres adultas, jovens, solteiras, que buscam atendimento psicológico espontaneamente, por distúrbios de ordem afetiva e de relacionamento (Carvalho \& Telles, 2001; Enéas, Faleiros \& Sá, 2000; Hahn \& Ferraz, 1998; Santos, Moura, Pasian \& Ribeiro, 1993; Terzis \& Carvalho, 1986).

O estado civil da clientela é disposto na Tabela 4

Distribuição dos Pacientes por Estado Civil da Clientela de Clínicas-Escola de Cursos de Psicologia da Região Metropolitana de Porto Alegre

\begin{tabular}{lllllllllll}
\hline & $\mathrm{A}$ & $\mathrm{B}^{2}$ & $\mathrm{C}$ & $\mathrm{D}$ & $\mathrm{E}$ & $\mathrm{F}$ & $\mathrm{G}$ & $\mathrm{H}$ & Total & $\%$ \\
\hline Até 18 anos & 275 & - & 64 & 1 & 80 & 215 & 68 & 91 & 794 & $41,05 \%$ \\
Solteiro & 119 & - & 15 & 16 & 36 & 53 & 87 & 10 & 336 & $17,37 \%$ \\
Casado/União estável & 98 & - & 23 & 4 & 26 & 125 & 56 & 20 & 352 & $18,20 \%$ \\
Separado/ Divorciado & 41 & - & 4 & 4 & 26 & 10 & 12 & 2 & 99 & $5,12 \%$ \\
Viúvo & 8 & - & 3 & 0 & 9 & 2 & 1 & 1 & 24 & $1,24 \%$ \\
Não consta & $\mathrm{O}$ & - & 91 & 20 & $\mathrm{O}$ & 142 & 75 & 1 & 329 & $17,01 \%$ \\
\hline Total & 541 & - & 200 & 45 & 177 & 547 & 299 & 125 & 1934 & $100,00 \%$ \\
\hline
\end{tabular}

${ }^{2}$ Alguns dados relativos à $\mathrm{C}$-e $B$ (estado civil, renda familiar e ocupação) não são registrados pela equipe da mesma for- ma que o investigado nesta pesquisa, não sendo possível incluí-los para este estudo. 
No que diz respeito ao estado civil da clientela, apresentado na Tabela 4, constata-se o elevado percentual de sujeitos de até 18 anos (41,05\%; 794 sujeitos). Foi optado por desconsiderar o estado civil destes sujeitos devido ao fato de se tratarem predominantemente de crianças. Estes dados são esperados, se considerarmos também as informações fornecidas pela Tabela 2, que descreve uma população predominantemente jovem.

Quanto à população adulta, foram encontrados 17,37\% (336 sujeitos) solteiros, seguidos, pelos 18,208\% (352 sujeitos) casados ou em união estável, 5,12\% (99 sujeitos) separados ou divorciados e 1,24\% (24 sujeitos) viúvos.

Quando pesquisaram a população adulta das C-e, Terzis e Carvalho (1998), em pesquisa realizada na Pontifícia Universidade Católica de Campinas (PUCCamp), encon- traram $54,8 \%$ solteiros, $40,7 \%$ casados e amasiados, $3,3 \%$ separados e 1,2\% viúvo. Santos et al. (1993) realizaram pesquisa no Centro de Psicologia Aplicada (CPA) da Faculdade de Filosofia, Ciências e Letras de Ribeirão Preto da Universidade de São Paulo (FFCLRP-USP), analisando prontuários de pacientes a partir de 13 anos de idade, atendidos no período de 1987 e 1989 e encontraram 64\% solteiros, $27 \%$ casados, $4 \%$ separados, $2,5 \%$ viúvos e $3 \%$ não consta. Nestas duas pesquisas, a maioria da população é de solteiros, diferente do encontrado na Região Metropolitana de Porto Alegre. Todavia, a quantidade de informações faltantes (17,01\%, 329 sujeitos) pode prejudicar este resultado.

A distribuição dos pacientes por renda familiar é apresentada na Tabela 5:

Tabela 5

Distribuição dos Pacientes por Faixa de Renda Familiar da Clientela das Clínicas-Escola de Cursos de Psicologia da Região Metropolitana de Porto Alegre

\begin{tabular}{|c|c|c|c|c|c|c|c|c|c|c|}
\hline & $\mathrm{A}$ & $\mathrm{B}$ & $\mathrm{C}$ & $\mathrm{D}$ & $\mathrm{E}$ & $\mathrm{F}$ & G & $\mathrm{H}$ & Total & $\%$ \\
\hline Até $\mathrm{R} \$ 250,00$ & 46 & - & 4 & 7 & 9 & 33 & 7 & 8 & 114 & 5,89 \\
\hline $\mathrm{R} \$ 251-500,00$ & 133 & - & 16 & 7 & 71 & 126 & 41 & 39 & 433 & 22,39 \\
\hline $\mathrm{R} \$ 501-1000,00$ & 162 & - & 35 & 5 & 71 & 143 & 52 & 49 & 517 & 26,73 \\
\hline $\mathrm{R} \$ 1001-2000,00$ & 74 & - & 15 & 4 & 26 & 47 & 28 & 8 & 202 & 10,44 \\
\hline $\mathrm{R} \$ 2001-3000,00$ & 11 & - & 1 & $\mathrm{O}$ & $\mathrm{O}$ & 10 & 3 & 1 & 26 & 1,34 \\
\hline $\mathrm{R} \$ 3001-5000,00$ & 4 & - & 2 & $\mathrm{O}$ & $\mathrm{O}$ & 5 & 3 & 1 & 15 & 0,78 \\
\hline Acima de $\mathrm{R} \$ 5000,00$ & 1 & - & 2 & $\mathrm{O}$ & $\mathrm{O}$ & $\mathrm{O}$ & $\mathrm{O}$ & $\mathrm{O}$ & 3 & 0,16 \\
\hline Não consta & 110 & - & 125 & 22 & $\mathrm{O}$ & 183 & 165 & 19 & 624 & 32,26 \\
\hline Total & 541 & - & 200 & 45 & 177 & 547 & 299 & 125 & 1934 & $100 \%$ \\
\hline
\end{tabular}

A Tabela 5 demonstra que as freqüências maiores de renda familiar são das faixas de $\mathrm{R} \$ 501$ a $\mathrm{R} \$ 1000$, com $26,73 \%$ (517 sujeitos) e de $\mathrm{R} \$ 251$ a $\mathrm{R} \$ 500$, com $22,39 \%$ (433 pacientes). Se somarmos as três primeiras faixas salariais, percebemos que $55,01 \%$ (1064 sujeitos) possuem renda familiar de até $\mathrm{R} \$ 1000$. O percentual de sujeitos diminui significativamente à medida que a renda aumenta. Este dado vai ao encontro de outras pesquisas sobre descrição da população atendida em C-e, pois há um consenso de que toda a clientela deste tipo de clínica é economicamente menos favorecida (Ancona-Lopez, 1983b).
$\mathrm{Na}$ Tabela 5 ainda é importante verificar que não consta a renda de 32,26\% (624 sujeitos) da clientela, que é o dado mais prejudicado desta pesquisa, em relação às informações sociodemográficas faltantes. Isto pode ser ocasionado tanto por uma dificuldade de registro das equipes das C-e como pela impossibilidade de os pacientes informarem sobre este dado a respeito de sua família; entretanto esta grande quantidade de informações faltantes prejudica uma adequada descrição do nível socioeconômico da clientela.

O nível de escolaridade é apresentado na Tabela 6: 
Tabela 6

Distribuição dos Pacientes por Nível de Escolaridade da Clientela das Clínicas-Escola de Cursos de Psicologia da Região Metropolitana de Porto Alegre

\begin{tabular}{lllllllllll}
\hline & $\mathrm{A}$ & $\mathrm{B}$ & $\mathrm{C}$ & $\mathrm{D}$ & $\mathrm{E}$ & $\mathrm{F}$ & $\mathrm{G}$ & $\mathrm{H}$ & Total & $\%$ \\
\hline Adulto analfabeto & $\mathrm{O}$ & 9 & $\mathrm{O}$ & $\mathrm{O}$ & $\mathrm{O}$ & $\mathrm{O}$ & 3 & $\mathrm{O}$ & 12 & $\mathrm{O}, 36$ \\
Criança/adolescente & & & & & & & & & & \\
não freqüenta Escola & 16 & $\mathrm{O}$ & $\mathrm{O}$ & $\mathrm{O}$ & $\mathrm{O}$ & 6 & 2 & 8 & 32 & 0,95 \\
Escola Especial & 1 & 10 & $\mathrm{O}$ & $\mathrm{O}$ & $\mathrm{O}$ & $\mathrm{O}$ & 1 & $\mathrm{O}$ & 12 & 0,36 \\
Escola Infantil & 29 & 72 & 41 & 2 & 80 & 9 & 7 & 7 & 247 & 7,34 \\
Ensino Fundamental & 229 & 557 & 18 & 17 & 44 & 225 & 71 & 84 & 1245 & 37,00 \\
Ensino Médio & 131 & 388 & 27 & 7 & 53 & 73 & 77 & 21 & 777 & 23,09 \\
Ensino Superior & 71 & 267 & 10 & $\mathrm{O}$ & $\mathrm{O}$ & 45 & 97 & 2 & 485 & 14,41 \\
Pós-graduação & 5 & 6 & $\mathrm{O}$ & $\mathrm{O}$ & $\mathrm{O}$ & $\mathrm{O}$ & $\mathrm{O}$ & $\mathrm{O}$ & 11 & 0,33 \\
Não consta & 59 & 129 & 104 & 19 & $\mathrm{O}$ & 189 & 48 & 3 & 551 & 16,37 \\
\hline Total & 541 & 1438 & 200 & 45 & 177 & 547 & 299 & 125 & 3372 & $100 \%$ \\
\hline
\end{tabular}

O nível de escolaridade (Tabela 6) também está de acordo com a faixa etária predominante da população. Esperase que crianças e adolescentes estejam cursando Ensino Fundamental e Médio, os quais são os níveis de escolaridade mais freqüentes, com 37,00\% (1245 sujeitos) e 23,09\% (777 sujeitos), respectivamente. Este dado também é coerente com as faixas de renda familiar dos pacientes, pois é provável que famílias que se mantenham com uma renda baixa possuam baixos níveis de escolarização.

Os índices de pacientes que cursam Ensino Superior (14,41\%, com 485 sujeitos) ou Pós-Graduação (O,33\%, com 11 sujeitos) podem ser explicados por uma demanda interna das próprias universidades em que as C-e estão inseridas, visto que a maioria das C-e participantes da pesquisa $(A, B, C, D, E, G$ e $H$ ) encontra-se localizada nos campi universitários. Isso demonstra que as C-e parecem estar atendendo também a demandas internas das universidades, em que os próprios alunos e funcionários podem usufruir dos atendimentos prestados pela C-e, usualmente descrita como mais utilizada pela comunidade externa. Essa atividade é descrita por R. S. Peres, Santos e Coelho (2004) como fundamental tanto para a C-e como para os estagiários que ali exercem suas práticas, no sentido de não restringir a possibilidade de atendimento nem direcionar suas estratégias de intervenção a uma população específica, favorecendo a diversidade.

Romaro e Capitão (2003), em pesquisa realizada na universidade de São Francisco entre 1995 e 2000, encontraram resultados semelhantes, em que $45,1 \%$ dos registros são relativos ao $1^{\circ}$ Grau Incompleto, $14,6 \%$ ao $2^{\circ}$ Grau e $15,2 \%$ ao Curso Superior Incompleto.

A distribuição dos pacientes por ocupação é apresentada na Tabela 7 :

Tabela 7

Distribuição dos Pacientes por Ocupação da Clientela das Clínicas-Escola de Cursos de Psicologia da Região Metropolitana de Porto Alegre

\begin{tabular}{|c|c|c|c|c|c|c|c|c|c|c|}
\hline & $\mathrm{A}$ & $\mathrm{B}$ & $\mathrm{C}$ & $\mathrm{D}$ & $\mathrm{E}$ & $\mathrm{F}$ & G & $\mathrm{H}$ & Total & $\%$ \\
\hline Estudante & 297 & - & 55 & 15 & 81 & 180 & 100 & 93 & 821 & 42,45 \\
\hline Não manuais baixo padrão & 59 & - & 4 & 4 & 24 & 54 & 38 & 7 & 91 & 4,71 \\
\hline Não manuais médio padrão & 28 & - & 7 & $\mathrm{O}$ & 27 & 21 & 75 & 8 & 181 & 9,36 \\
\hline Não trabalha & 37 & - & 1 & 4 & 26 & 30 & 19 & 1 & 60 & 3,10 \\
\hline Do lar & 16 & - & 1 & 2 & 10 & 17 & 19 & 6 & 29 & 1,50 \\
\hline Liberais/ administradores & 21 & - & $\mathrm{O}$ & $\mathrm{O}$ & $\mathrm{O}$ & 19 & 13 & 8 & 23 & 1,19 \\
\hline Habilidades manuais & 18 & - & $\mathrm{O}$ & $\mathrm{O}$ & 9 & 5 & 6 & 1 & 90 & 4,65 \\
\hline Aposentado & 12 & - & 3 & $\mathrm{O}$ & $\mathrm{O}$ & 11 & 9 & $\mathrm{O}$ & 154 & 7,96 \\
\hline Funcionário Público & 3 & - & $\mathrm{O}$ & $\mathrm{O}$ & $\mathrm{O}$ & 5 & 5 & $\mathrm{O}$ & 13 & 0,67 \\
\hline Dirigentes/executivos & $\mathrm{O}$ & - & $\mathrm{O}$ & $\mathrm{O}$ & $\mathrm{O}$ & 4 & $\mathrm{O}$ & $\mathrm{O}$ & 32 & 1,65 \\
\hline Não manuais alto padrão & $\mathrm{O}$ & - & 1 & $\mathrm{O}$ & $\mathrm{O}$ & 1 & $\mathrm{O}$ & 1 & 27 & 1,40 \\
\hline Não consta & 50 & - & 128 & 20 & $\mathrm{O}$ & 200 & 15 & $\mathrm{O}$ & 413 & 21,35 \\
\hline Total & 541 & - & 200 & 45 & 177 & 547 & 299 & 125 & 1934 & $100 \%$ \\
\hline
\end{tabular}


Tratando-se de uma população predominantemente jovem, os registros quanto à ocupação (Tabela 7) encontram-se de acordo. A maior concentração da população, $42,45 \%$ (821 sujeitos) é estudante. Resultados semelhantes foram encontrados por Terzis e Carvalho (1988), que descreveram 44,1\% da população investigada como estudantes; e por Santos et al. (1993), que relatam que 33\% da população investigada somente estudam.

Trabalhos não manuais de baixo padrão, tais como au- xiliares de escritórios e trabalhadores em serviços gerais representam 9,36\% (181 sujeitos), dados que também podem contribuir para explicar a renda familiar da clientela (Tabela 5). Esses achados estão de acordo com os 15\% de trabalhadores não-manuais de baixo padrão encontrados por Santos et al. (1993). As demais modalidades de ocupação declaradas encontram-se distribuídas em pequenas faixas de agrupamento.

$\mathrm{Na}$ Tabela 8 estão dispostos os locais que encaminham a clientela às C-e:

Tabela 8

Distribuição dos Pacientes por Fonte de Encaminhamento da Clientela das Clínicas-Escola de Cursos de Psicologia da Região Metropolitana de Porto Alegre

\begin{tabular}{|c|c|c|c|c|c|c|c|c|c|c|}
\hline & $\mathrm{A}$ & B & $\mathrm{C}$ & $\mathrm{D}$ & $\mathrm{E}$ & $\mathrm{F}$ & G & $\mathrm{H}$ & Total & $\%$ \\
\hline Não consta & 98 & 366 & 97 & 19 & $\mathrm{O}$ & 136 & 56 & 3 & 775 & 22,98 \\
\hline Instituições Escolares & 48 & 214 & 13 & 10 & 62 & 57 & 21 & 49 & 474 & 14,06 \\
\hline Busca espontânea & 126 & 72 & 59 & 2 & 19 & 43 & 93 & 5 & 419 & 12,43 \\
\hline Conhecido & 24 & 195 & 3 & 4 & 53 & 34 & 28 & 16 & 357 & 10,59 \\
\hline Médicos & 73 & 109 & 9 & $\mathrm{O}$ & $\mathrm{O}$ & 90 & 14 & 9 & 304 & 9,02 \\
\hline Familiar & 72 & 79 & $\mathrm{O}$ & 1 & 16 & 25 & 21 & 19 & 233 & 6,91 \\
\hline Postos de Saúde & 11 & 141 & $\mathrm{O}$ & 3 & $\mathrm{O}$ & 33 & 2 & 2 & 192 & 5,69 \\
\hline Outros & 24 & 53 & $\mathrm{O}$ & 3 & 27 & 49 & 27 & 6 & 189 & 5,60 \\
\hline Serviços de psicologia & 18 & 89 & $\mathrm{O}$ & 2 & $\mathrm{O}$ & 19 & 15 & 3 & 146 & 4,33 \\
\hline Hospitais & 25 & 76 & 11 & 1 & $\mathrm{O}$ & 4 & $\mathrm{O}$ & $\mathrm{O}$ & 117 & 3,47 \\
\hline Assistente Social & 14 & 5 & 1 & $\mathrm{O}$ & $\mathrm{O}$ & 38 & $\mathrm{O}$ & 2 & 60 & 1,78 \\
\hline Paciente da C-e & 4 & 32 & $\mathrm{O}$ & $\mathrm{O}$ & $\mathrm{O}$ & 14 & 4 & 4 & 58 & 1,72 \\
\hline Fonoaudiólogo & $\mathrm{O}$ & 6 & 7 & $\mathrm{O}$ & $\mathrm{O}$ & 3 & 11 & $\mathrm{O}$ & 27 & 0,80 \\
\hline Rádio/jornal/TV/Internet & 4 & 1 & $\mathrm{O}$ & $\mathrm{O}$ & $\mathrm{O}$ & 2 & 7 & $\mathrm{O}$ & 14 & 0,42 \\
\hline Total & 541 & 1438 & 200 & 45 & 177 & 547 & 299 & 125 & 3372 & $100 \%$ \\
\hline
\end{tabular}

$\mathrm{Na}$ Tabela 8, verifica-se que as instituições escolares representam 14,06\% (447 pacientes). Em pesquisa realizada na C-e do Instituto de Psicologia da USP, Silvares (1993) constatou que pais mais escolarizados, de nível superior, encaminham seus filhos à clínica em idade mais precoce do que os menos escolarizados. Os pais pouco escolarizados atribuem este papel à escola, precisando de um intermediário para identificar a problemática infantil. Este intermediário, por sua vez, tende a ser mais seletivo nessa identificação e a encaminhar as crianças mais por problemas escolares. Para a autora, há a necessidade de se promover programas preventivos, tornando-se mais eficaz que a clínica vá até estas crianças com programas profiláticos do que aguardar sua vinda à clínica, após anos de repetência escolar.
Na revisão de artigos, verifica-se que as fontes de encaminhamento se centram nas escolas quando falamos de população infantil, enquanto adolescentes e adultos apresentam procura espontânea por auxílio psicológico (Ancona-Lopez, 1983a; Arcaro, 1991; Marturano, Magna \& Mutha, 1993).

A segunda maior freqüência disposta na tabela acima é representada por $12,43 \%$ da clientela (419 sujeitos), que declararam ter buscado atendimento psicológico espontaneamente. Conhecidos (10,59\%, com 357 sujeitos), médi$\cos (9,02 \%$, com 304 sujeitos) e familiares $(6,91 \%$, com 233 sujeitos) também podem ser mencionados entre as fontes que mais encaminham pacientes.

Observam-se na Tabela 9 as queixas principais citadas pelos pacientes como motivo da busca por atendimento. 
Tabela 9

Distribuição dos Pacientes por Queixa Apresentada (motivo da busca de atendimento) da Clientela das Clinicas-Escola de Cursos de Psicologia da Região Metropolitana de Porto Alegre

\begin{tabular}{|c|c|c|c|c|c|c|c|c|c|c|}
\hline & $\mathrm{A}$ & $\mathrm{B}$ & $\mathrm{C}$ & $\mathrm{D}$ & $\mathrm{E}$ & $\mathrm{F}$ & G & $\mathrm{H}$ & Total & $\%$ \\
\hline \multicolumn{11}{|l|}{ Dificuldades no } \\
\hline Comportamento afetivo & 263 & 189 & 62 & 12 & 72 & 231 & 52 & 59 & 940 & 27,88 \\
\hline Dificuldades em & & & & & & & & & & \\
\hline Processos cognitivos & 52 & 602 & 20 & 4 & 40 & 50 & 14 & 26 & 808 & 23,96 \\
\hline \multicolumn{11}{|l|}{ Dificuldades de } \\
\hline Relacionamento interpessoal & 41 & 318 & 10 & 2 & 56 & 30 & 18 & 15 & 490 & 14,53 \\
\hline Distúrbios Psiquiátricos & 20 & 64 & 3 & $\mathrm{O}$ & $\mathrm{O}$ & 22 & 15 & 3 & 127 & 3,77 \\
\hline \multicolumn{11}{|l|}{ Dificuldades na Profissão/ } \\
\hline Trabalho & 3 & 37 & 1 & 1 & o & 3 & 69 & $\mathrm{o}$ & 114 & 3,38 \\
\hline Distúrbios Orgânicos & 15 & 49 & 4 & $\mathrm{O}$ & $\mathrm{O}$ & 9 & 22 & 5 & 104 & 3,08 \\
\hline \multicolumn{11}{|l|}{ Distúrbios de alimentação/ } \\
\hline Sintomas físicos & 23 & o & 4 & $\mathrm{O}$ & $\mathrm{O}$ & 22 & 1 & 1 & 51 & 1,51 \\
\hline \multicolumn{11}{|l|}{ Dependência química/ } \\
\hline Adições & 5 & 24 & 1 & $\mathrm{O}$ & $\mathrm{O}$ & 14 & o & 2 & 46 & 1,36 \\
\hline Dificuldades na vida diária & 7 & 6 & 20 & 6 & $\mathrm{O}$ & 12 & 1 & 2 & 36 & 1,07 \\
\hline Dificuldades na esfera sexual & 7 & 17 & 1 & $\mathrm{O}$ & $\mathrm{O}$ & 7 & 1 & 92 & 125 & 3,71 \\
\hline Queixas de terceiros & 3 & o & $\mathrm{o}$ & $\mathrm{O}$ & $\mathrm{O}$ & 22 & 1 & 4 & 30 & 0,89 \\
\hline Outras & 1 & 23 & $\mathrm{O}$ & $\mathrm{O}$ & $\mathrm{O}$ & $\mathrm{O}$ & 2 & 1 & 27 & 0,80 \\
\hline Não consta & 92 & 94 & 89 & 20 & 0 & 108 & 99 & 3 & 505 & 14,98 \\
\hline Total & 541 & 1438 & 200 & 4.5 & 177 & 547 & 299 & 125 & 3372 & $100 \%$ \\
\hline
\end{tabular}

Na Tabela 9, constata-se que as dificuldades no comportamento afetivo são citadas por $27,88 \%$ dos sujeitos (940 pacientes). Este tipo de queixa inclui, principalmente, agressividade, ansiedade, isolamento social, depressão, choro freqüente, dependência, imaturidade e temores. Também é possível observar, como destaque entre os motivos de busca por atendimento, as dificuldades em processos cognitivos, com 23,96\% (808 sujeitos), que incluem, principalmente, as dificuldades de aprendizagem. A seguir, as dificuldades de relacionamento interpessoal também são relatadas na freqüência de $14,53 \%$ dos pacientes (490 sujeitos). Esses dados são similares aos que Wolf (1988) encontrou pesquisando 956 prontuários da C-e da Universidade Federal de São Paulo (UNIFESP) entre 1978 e 1981. Como razão pela busca por atendimento psicológi- co, $24 \%$ referiram distúrbios de escolaridade, $27,4 \%$ distúrbios afetivo-emocionais e 14,9\% distúrbios da sociabilidade.

De um modo geral, os achados desta pesquisa estão de acordo com a revisão da bibliografia realizada por Levandowski (1998), a qual refere que, quanto ao motivo e à fonte de encaminhamento, na população infantil, são característicos os problemas de aprendizagem ou relacionados ao contexto escolar, enquanto a população de adolescentes e adultos apresenta como queixa problemas afetivos e de ordem funcional, sendo saliente em adultos problemas de ordem afetiva e alcoolismo.

$\mathrm{Na}$ Tabela 10 está descrita a situação atual dos pacientes que buscaram atendimento nas C-e. 
Campezatto, P. V. M. \& Nunes, M. L. T., (2007). Caracterização da Clientela das Clínicas-Escola de Cursos de Psicologia da Região Metropolitana de Porto Alegre.

Tabela 10

Distribuição dos Pacientes por Situação Atual da Clientela das Clínicas-Escola de Cursos de Psicologia da Região Metropolitana de Porto Alegre

\begin{tabular}{|c|c|c|c|c|c|c|c|c|c|c|}
\hline & $\mathrm{A}$ & B & $\mathrm{C}$ & $\mathrm{D}$ & $\mathrm{E}$ & $\mathrm{F}$ & G & $\mathrm{H}$ & Total & $\%$ \\
\hline $\begin{array}{l}\text { Buscaram } \\
\text { atendimento }\end{array}$ & 541 & 1438 & 200 & 45 & 177 & 547 & 299 & 125 & 3372 & $100 \%$ \\
\hline $\begin{array}{l}\text { Foram } \\
\text { re-encaminhados }\end{array}$ & 296 & 19 & 10 & 11 & 14 & 19 & 7 & 26 & 402 & $11,92 \%$ \\
\hline $\begin{array}{l}\text { Receberam atendimento/ } \\
\text { aguardam em lista de espera }\end{array}$ & 193 & 470 & 190 & 15 & 136 & 424 & 292 & 99 & 1819 & $53,94 \%$ \\
\hline Não consta & 52 & 949 & $\mathrm{O}$ & 19 & 27 & 104 & $\mathrm{O}$ & 6 & 1157 & $34,31 \%$ \\
\hline
\end{tabular}

A partir dos dados apresentados na Tabela 10, constatase que, dos 3247 pacientes que buscaram atendimento, $11,92 \%$ (402 sujeitos) foram re-encaminhados. O número relativamente grande de encaminhamentos para outros profissionais remonta à necessidade de trabalho multiprofissional (Wolf, 1988), o que ocorre em poucas das C-e participantes da pesquisa. 52,97\% (1729 sujeitos) receberam atendimento ou aguardam em lista de espera.
Yehia (1994) lembra que a redução do tempo em lista de espera favorece maior eficiência do trabalho realizado. Um número muito elevado de informações faltantes (34,13\%, relativo a 1151 sujeitos) pode comprometer os resultados desta tabela.

A Tabela 11 descreve a situação final dos atendimentos realizados no ano de 2004 pelas C-e participantes desta pesquisa.

Tabela 11

Distribuição dos Pacientes por Situação Final dos Atendimentos da Clientela das Clínicas-Escola de Cursos de Psicologia da Região Metropolitana de Porto Alegre

\begin{tabular}{|c|c|c|c|c|c|c|c|c|c|c|}
\hline & $\mathrm{A}$ & $\mathrm{B}$ & $\mathrm{C}$ & $\mathrm{D}$ & $\mathrm{E}$ & $\mathrm{F}$ & G & $\mathrm{H}$ & Total & $\%$ \\
\hline Receberam atendimento & 193 & 470 & 190 & 15 & 136 & 424 & 284 & 99 & 1811 & $100,00 \%$ \\
\hline $\begin{array}{l}\text { Abandonaram } \\
\text { os atendimentos }\end{array}$ & 23 & 322 & 39 & 1 & 27 & 185 & 74 & 21 & 692 & $38,21 \%$ \\
\hline Alta & 6 & 21 & 23 & 14 & 109 & 60 & 29 & 24 & 286 & $15,79 \%$ \\
\hline $\begin{array}{l}\text { Em atendimento/ } \\
\text { em lista de espera }\end{array}$ & 164 & 146 & 128 & $\mathrm{O}$ & $\mathrm{O}$ & 179 & 181 & 44 & 842 & $46,49 \%$ \\
\hline
\end{tabular}

Segundo os dados da Tabela 11, 1811 pacientes receberam atendimento psicológico nas $\mathrm{C}$-e, mas este dado possivelmente está comprometido devido às informações faltantes na Tabela 9. Não foi possível informar se 34,31\% (1157 sujeitos) permaneceram em atendimento nas C-e ou foram encaminhados para outras instituições de saúde ou de atendimento psicológico. Dos 1811 pacientes que é possível afirmar que receberam atendimento nestas C-e, $38,21 \%$ (692 sujeitos) abandonaram o tratamento, dado esperado com relação à literatura. A C-e $G$ afirma observar que estes abandonos ocorrem, em sua maioria, durante o período de avaliação. Índices de pacientes abandonantes situam-se entre $30 \%$ a $60 \%$, e o período crucial para os abandonos se dá em torno da $6^{\mathrm{a}}$ sessão. Desse modo, observa-se que a fase inicial da Psicoterapia é a mais delicada para a continuação do tratamento e os níveis de abandonos não se elevam muito depois dessa etapa (Lhullier, 2002). Wolf (1988) relata um índice de 24,04\% de aban- donos e desistências, durante a triagem, na C-e pesquisada.

Yehia (1994), ao descrever a população que busca o serviço de atendimento de superdotados em uma clínica psicológica, aponta que $34 \%$ desistem na triagem. Acredita que a forma como acontece a triagem favorece este alto nível de desistência, uma vez que o triador já oferece indicações a respeito das possibilidades de estimulação e dos recursos oferecidos pela comunidade, satisfazendo a clientela com os esclarecimentos recebidos. Talvez por isso, Ancona-Lopez (1983b) descreva que o paciente conclui a triagem, mas não comparece aos atendimentos, o que ocorreu com $31,1 \%$ da sua amostra.

Os 15,79\% (286 sujeitos) dos pacientes que concluíram os atendimentos com alta representam um percentual elevado, comparando-se aos 4,6\% escritos por Ancona-Lopez (1983b). Não é possível afirmar quantos pacientes estavam aguardando por atendimentos em lista de espera, nem se o desfecho destes atendimentos será ou não favorável. 
Mesmo assim, o índice de conclusão dos atendimentos com a concordância entre as partes (paciente e terapeuta) foi elevado. Também é necessário considerar que nas C-e participantes deste estudo também fazem parte de sua equipe profissionais já formados, como contratados ou exercendo práticas de Pós-Graduação, e não apenas alunos de graduação em Psicologia.

\section{Considerações Finais}

Ao final deste estudo, algumas considerações podem ser feitas no que diz respeito à caracterização da clientela das C-e dos cursos de Psicologia da Região Metropolitana de Porto Alegre.

Em primeiro lugar, é possível reconhecer a similaridade dos achados com muitos dos estudos oriundos de outras regiões do Brasil, permitindo-se a inferência de dois perfis típicos do paciente que busca atendimento em C-e no Brasil: crianças, do sexo masculino, encaminhadas por escolas devido a dificuldades de aprendizagem e comportamento e mulheres jovens, que buscam atendimento espontaneamente devido a conflitos relativos ao comportamento afetivo. A grande maioria desta clientela dificilmente teria acesso aos consultórios privados de psicólogos clínicos devido à situação socioeconômica das famílias em que estão inseridas.

O primeiro perfil, que descreve crianças de 6 a 10 anos de idade, corresponde ao período de escolarização, situação que tende a trazer maiores conflitivas em meninos do que em meninas. As queixas relativas a processos cognitivos, que remetem a dificuldades de aprendizagem, são mais comuns nos meninos do que nas meninas de mesma faixa etária. As meninas e, principalmente, as mulheres jovens parecem ter mais dificuldades de ordem emocional como motivo de busca por atendimento, sendo comuns as crises de choro, tristeza, ansiedade. Pacientes do sexo feminino também buscam atendimento psicológico espontaneamente, devido a conflitos de relacionamento interpessoal, queixa pouco expressa por homens.

Todavia, conhecimentos de Psicologia do Desenvolvimento e Psicopatologia não se mostram capazes de explicar os motivos pelos quais tais pacientes são encaminhados com maior freqüência às C-e. A demanda de Psicoterapia que surge do próprio sujeito, no caso das mulheres adultas, faz sentido se for compreendido que a Psicoterapia é um espaço para a auto-reflexão e autoconhecimento, além de proporcionar a revisão de padrões de funcionamento desadaptativos.

Entretanto, quando se trata de crianças escolares como população típica de serviços de atendimento, com encaminhamentos maciços por escolas, é necessário discriminar os motivos de encaminhamento que são ou não de ordem psicológica. A entrada na escola gera angústias em muitas crianças, que se deparam, principalmente, com conflitos relativos à capacidade simbólica, à separação e à imaturidade para a aprendizagem formal, os quais são de natureza emocional, mas eclodem comumente na etapa de educação formal (Castro \& Nunes, 1999). Mas também as escolas ou as próprias equipes responsáveis pelo ensino de crianças nesta faixa etária podem expressar conflitivas próprias com a tarefa do ensino através das crianças, atribuindo-lhes as próprias dificuldades ou designando-as como porta-vozes (Boarini \& Borges, 1998). Há, também, a questão do "ajustamento" à escola e aos padrões de ensino, em que o desvio da norma pode tomar proporções assustadoras para quem lida com a educação. É sabido, ainda, que meninos tendem a ser menos contidos do que meninas a expressar suas dificuldades por meio de comportamentos devido a motivos culturais e sociais, o que não quer dizer que meninas sofram menos tais conflitos (Castro \& Nunes, 1999).

A partir disso, deve-se refletir sobre a atribuição de papéis às C-e e às equipes de atendimento, já que muitas vezes estas recebem demandas que não lhes pertencem, pois não têm indicação para Psicoterapia (Ancona-Lopez, 1983b). Para realizar tal indicação, é necessária capacidade de discriminar adequadamente os casos que devem ou não receber atendimento em C-e, o que pressupõe uma formação profissional coesa. Neste sentido, o (futuro) profissional deve estar apto a recorrer a outras áreas de atuação da própria Psicologia, como a Escolar, Educacional, Institucional, Social ou Organizacional, bem como a outras áreas relacionadas à saúde e que visem ao bem-estar das pessoas, não aceitando indiscriminadamente a clientela que chega às C-e. Tais aspectos remetem à dupla função da C-e, que deve atender adequadamente às necessidades dos que a buscam, seguindo a ética profissional, proporcionando uma formação adequada ao acadêmico.

Uma forma de atender a estas outras demandas seria o deslocamento de profissionais e estagiários às comunidades, capacitando-os pessoal e profissionalmente para a realização de trabalho preventivo, o que atenderia às necessidades diversas e auxiliaria na discriminação, por parte do profissional/estagiário, de casos que devem ou não receber atendimento psicoterapêutico na própria C-e. Estas estratégias também foram adotadas em C-e de outras regiões do país, como medidas de adequação de suas técnicas à população atendida a partir de suas próprias avaliações diagnósticas (Guerrelhas \& Silvares, 2000; Silvares, 1993, 2000).

Um outro método de atender a estas e a outras demandas das C-e seria uma terceira função que a C-e tem executado, ainda não adotada na literatura: aliada ao ensino e ao atendimento, a execução da função de ser fonte e estímulo para o desenvolvimento de pesquisa, o que propicia qualificação do estagiário, fornece feedback à C-e, e beneficia sua clientela.

No que diz respeito a esta pesquisa, ainda é necessário destacar a falta de registros adequados em grande parte das instituições de atendimento clínico em Psicologia e a dificuldade em realizar pesquisas diante da falta de dados. Isso pode ser verificado pelo tempo de demora em se obter os dados das C-e, devido à necessidade de realizar pesquisas internas para coletar as informações solicitadas e, também, pela necessidade de auxílio que algumas C-e requisitaram às pesquisadoras na construção do banco de 
dados. Essas dificuldades denotam, sobretudo, o quanto realizar pesquisas em instituições de atendimento psicológico e clínico não é usual. A falta da vertente 'pesquisa' pode comprometer, inclusive, a formação do aluno, já que não obtém feedback da qualidade dos atendimentos e não estimula esta atividade como papel do psicólogo clínico. Assim, este trabalho também contribuiu com tais aspectos ao auxiliar na coleta dos dados das C-e e ao instigar pesquisas com seus materiais.

As informações faltantes, retratadas como 'Não consta' nas tabelas também devem ser consideradas como fator comprometedor da qualidade dos dados de algumas variáveis desta pesquisa, pois atingiram percentuais muito significativos, variando de 5,36\% (na variável 'sexo dos sujeitos') a 32,26\% (na variável 'renda familiar do sujeito').

Todavia, o retorno dos questionários foi acompanhado de relatos favoráveis dos próprios coordenadores/diretores de algumas C-e, pois a iniciativa da pesquisa trouxe benefícios para as próprias clínicas, que passaram a questionar o modo como realizam seus registros e a se organizarem de forma a facilitar pesquisas futuras, reconhecendo a sua importância.

Pode-se afirmar, no que concerne ao acolhimento da clientela que busca atendimento nas C-e da Região Metropolitana de Porto Alegre, que as triagens e a recepção dos pacientes estão sendo realizadas adequadamente, pois ocorre um percentual reduzido de re-encaminhamentos. A relevância social destas instituições torna-se evidente devido à grande quantidade de pacientes que buscaram por atendimento em 2004; demanda que pode crescer nos próximos anos de acordo com a estimativa do ponto de vista da epidemiologia, que prevê desordens mentais na infância em $23,2 \%$ das crianças, dos quais $10 \%$ são considerados casos moderados e severos, necessitando atendimento especializado, e 13,2\% são considerados casos leves ou duvidosos (Almeida Filho, 1985 citado por Boarini \& Borges, 1998), além das desordens mentais na vida adulta. Boa parte dessa população encontra na C-e uma das poucas possibilidades de receberem atendimento psicológico financeiramente acessível, sendo importante considerar a sugestão de Terzis e Carvalho (1988), no sentido de implementar estratégias preventivas nestes grupos populacionais que possam ser constituídos pelo perfil característico de cada C-e. Os índices de abandono e alta também são similares aos de outras clínicas de atendimento psicológico realizado por alunos de Graduação, Pós-Graduação ou de profissionais já formados, estando de acordo com o esperado pela revisão da literatura. Entretanto, tais índices considerados «esperados» pela literatura comprometem aproximadamente $38,21 \%$ dos atendimentos, o que deveria levar-nos a refletir constantemente sobre os motivos pelos quais os pacientes desistem dos atendimentos, com o intuito de aprimorar contínua e gradualmente a eficiência e qualidade dos serviços prestados, na tentativa de alcançar índices cada vez menores. Pode-se inferir que fatores como a demora para agendar uma primeira entrevista e o processo de espera para realizar Psicoterapia podem contribuir para a evasão.
Em decorrência dessas considerações, os resultados deste estudo se ampliam, favorecendo a realização de pesquisas futuras em C-e, que apresentam um material rico e mantêm as portas abertas para pesquisadores, possibilitando uma intersecção entre o ensino, a prática e a pesquisa. O Grupo de Pesquisa 'Avaliação e Intervenção em Psicoterapia e Psicossomática' da PUCRS é exemplo disso, em virtude de, com base nos resultados deste trabalho, estar dando seguimento a esta idéia, abrangendo a supervisão sob o ponto de vista do supervisionando, comparando alunos de Graduação e de Pós-Graduação (Saraiva \& Nunes, 2005), aprofundando os motivos de consultas de crianças quando buscam por atendimento psicológico em C-e no Rio Grande do Sul (Savalhia \& Nunes, 2005), avaliando crianças antes e depois da intervenção psicoterápica em C-e e comparando a evolução das conflitivas (Dian \& Nunes, 2005) e buscando mensurar a eficácia das psicoterapias, de orientação psicanalítica (Deakin \& Nunes, 2005).

\section{Referências}

Ancona-Lopez, M. A. (1983a). Características da clientela de clínicas-escola de Psicologia em São Paulo. Arquivos Brasileiros de Psicologia, 35(1), 78-92

Ancona-Lopez, M. A. (1983b). Considerações sobre o atendimento oferecido por clínicas-escola de Psicologia. Arquivos Brasileiros de Psicologia, 39(2), 123-135.

Arcaro, N. T. (1991). Investigação de aspectos da clientela e sistema de atendimento de um ambulatório da saúde mental Psicologia - USP, 2(1/2), 49-63.

Arcaro, N. T., \& Mejias, N. P. (1990). A evolução da assistência psicológica e em saúde mental: Do individual para o comunitário. Psicologia: Teoria e Pesquisa, 6(3), 25 1-266.

Barbosa, J. I. C., \& Silvares, E. F. M. (1994). Uma caracterização preliminar das clínicas-escola de Fortaleza. Estudos de Psicologia (Campinas), $11(3), 50-56$.

Bardin, L. (1988). Análise de conteúdo. Lisboa, Portugal: Edições 70.

Bernardes da Rosa, L. T., Garcia, R. M.; Domingos, N. A. M., \& Silvares, E. F. M. (2000). Caracterização do atendimento psicológico prestado por um serviço de Psicologia a crianças com dificuldades escolares. Estudos de Psicologia (Campinas), $17(3), 5-14$.

Boarini, M. L., \& Borges, R. F. (1998). Demanda infantil por serviços de saúde mental: Sinal de crise. Estudos de Psicologia (Natal), 3(1), 83-108

Calejon, L. M. C. (1995). Estudos com pacientes de clínica psicológica universitária. Mudanças - Psicoterapia e Estudos Psicossociais, 3(3/4), 235-254

Campezatto, P. V. M., \& Nunes, M. L. (2007). Atendimento em Clínicas-Escola de Psicologia da Região Metropolitana de Porto Alegre. Estudos de Psicologia (Campinas), 24(3), 363-374.

Capellini, S. A., Tonelotto, J. M. F., \& Ciasca, S. M. (2004). Medidas de desempenho escolar: Avaliação formal e opinião de professores. Estudos de Psicologia (Campinas), 21(2), 79-90.

Castro, M. G. K., \& Nunes, M. L. T. (1999). As percepções e memórias da psicoterapia vivida na infância: Um estudo de seguimento. Revista Brasileira de Psicoterapia, 1(2), 81-98.

Carvalho, M. J. C., \& Telles, S. R. A. (2001). Considerações sobre queixas de pacientes em triagens de clínica-escola. Psikhê, $6(1), 7-14$ 
Deakin, E. K., \& Nunes, M. L. T. (2005). Avaliação dos resultados da psicoterapia de orientação psicanalítica com crianças [Resumo]. In Anais da I Jornada de Intercâmbio Científico e Acadêmico da Psicologia na Contemporaneidade [CD-ROM]. Por to Alegre, RS: Pontifícia Universidade Católica do Rio Grande do Sul.

Dian, S. V., \& Nunes, M. L. T. (2005). Psicoterapia Psicanalítica com crianças: Avaliação dos conflitos através do Teste das Fábulas [Resumo]. In Anais da I Jornada de Intercâmbio Científico e Acadêmico da Psicologia na Contemporaneidade [CDROM]. Porto Alegre, RS: Pontifícia Universidade Católica do Rio Grande do Sul.

Enéas, M. L. E., Faleiros, J. C., \& Sá, A. C. A. (2000). Uso de psicoterapias breves em clínica-escola: Caracterização dos processos com adultos. Psicologia: Teoria e Prática, 2(2), 9-30.

Ferreira, T. (1998). Clínica e escola de Psicologia: Uma relação de extimidade. Psique (Belo Horizonte), 8(12), 38-45.

Ghazzi, M. S. (2000). Estimulação precoce: Características do trabalho nas APAEs do Rio Grande do Sul. Disser tação de Mestrado não-publicada, Pontifícia Universidade Católica do Rio Grande do Sul, Porto Alegre, RS.

Goodwin, J. C. (1995). Research in Psychology: Methods and design. New York: John Wiley.

Goldim, J. R. (1998). Psicoterapias e Bioética. In A. V. Cordioli (Ed.), Psicoterapias: Abordagens atuais (pp. 119-133). Por to Alegre, RS: Artes Médicas.

Graminha, S. S, V., \& Martins, M. A. O. (1993). Estudo das características da população que procura o serviço de atendimento infantil no centro de psicologia aplicada da FFCLRPUSP. Psico (Porto Alegre), 24(1), 119-130

Guerrelhas, F. F., \& Silvares, E. F. M. (2000). Grupos de espera recreativos: proposta para diminuir o índice de evasão em clínica-escola de psicologia. Temas em Psicologia da SBP, 8(3), 313-321.

Güntert, A. E. V. A., Camargo, C., Fabriani, C. B., Silva, S. M., Conti, J., Dias, C. C., Zanetti, F., \& Silva, T. C. (2000). As variáveis determinantes na aderência à psicoterapia: Uma investigação em clínica-escola. Psico USF, 5(2), 13-23.

Hahn, M. S., \& Ferraz, M. P. T. (1998). Características da clientela de um programa de saúde mental para estudantes universitários brasileiros. Revista ABP-APAL, 2O(2), 45-53.

Instituto Brasileiro de Geografia e Estatística. (2004). Retirado em 02 jul. 2004, de www.ibge.gov.br/cidadesat/ topwindow.htm?20

Lei $\mathrm{n}^{\circ} 4.119$ (1962). Dispõe sobre a formação em Psicologia e regulamenta a profissão de psicólogo. Capítulo IV, Artigo 16, p. 3. Retirado em 12 abr. 2004, de http://www.pol.org.br/ legislacao/pdf/lei_n_4t119.pdf

Levandowski, D. C. (1998). Caracterização da população atendida por clínicas-escola: Breve revisão da literatura nacional. Torre de Babel, 5(1/2), 87-110.

Lhullier, A. C. (2002). Abandono de tratamento em psicoterapias realizadas numa clínica-escola. Tese de Doutorado não-publicada, Pontifícia Universidade Católica do Rio Grande do Sul, Porto Alegre, RS.

Louzada, R. C. R. (2003). Caracterização da clientela atendida no Núcleo de Psicologia Aplicada da Universidade Federal do Espírito Santo. Estudos de Psicologia (Natal), 8(3), 45 1-457.

Marturano, E. M., Magna, J. M., \& Murtha, P. C. (1993). Procura de atendimento psicológico para crianças com dificuldades escolares: Um perfil da clientela. Psicologia: Teoria e Pesquisa, 9(1), 207-226.
Nunes, M. L. T., Campezatto, P. V. M., Cruxên, O. S., \& Savalhia J. A. D. (2005). Clínicas-Escola de Psicologia e Psicoterapia Psicanalítica: $\mathrm{O}$ duplo desafio de atender com qualidade à clientela e propiciar ao acadêmico uma boa formação. In B. G. Werlang \& M. da S. Oliveira (Eds.), Temas em Psicologia Clinica (pp. 29-35). São Paulo, SP: Casa do Psicólogo.

Peres, V. L. A. (1997). Triagem psicológica grupal: Procedimento e resultados obtidos com lista de espera de crianças, adolescentes e adultos, em uma clínica-escola de psicologia. Paidéia (12/13), 63-76.

Peres, R. S., Santos, M. A., \& Coelho, H. M. B. (2004). Perfil da clientela de um programa de pronto-atendimento a estudantes universitários. Psicologia em Estudo, 9(1), 47-54.

Romaro, R. A., \& Capitão, C. G. (2003). Caracterização da clientela da clínica-escola de Psicologia da Universidade de São Francisco. Psicologia: Teoria e Prática, 5(1), 111-121.

Santos, M. A., Moura, L., Pasian, S. R., \& Ribeiro, P. L. L. (1993). Caracterização da clientela de adolescentes e adultos de uma clínica-escola de Psicologia. Psicologia: Teoria e Pesquisa, 9(1), 123-144.

Saraiva, L. A., \& Nunes, M. L. T. (2005). A supervisão nas clínicas-escola e nos centros de formação em Psicoterapia psicanalítica [Resumo]. In Anais da I Jornada de Intercâmbio Científico e Acadêmico da Psicologia na Contemporaneidade [CDROM]. Por to Alegre, RS: Pontifícia Universidade Católica do Rio Grande do Sul

Savalhia J. A. D., \& Nunes, M. L. T. (2005). Motivos de consulta em crianças de clínicas-escola de cursos de Psicologia do interior do estado do Rio Grande do Sul [Resumo]. In Anais da I Jornada de Intercâmbio Científico e Acadêmico da Psicologia na Contemporaneidade [CD-ROM]. Porto Alegre, RS: Pontifícia Universidade Católica do Rio Grande do Sul.

Schmidt, M. L. S. (1992). Clínica-escola, escola da clínica? Boletim de Psicologia, 42(96/97), 99-103.

Silvares, E. F. M. (1993). O papel preventivo das clínicas-escola de Psicologia em seu atendimento a crianças. Temas em Psicologia, 2, 87-97.

Silvares, E. F. M. (1996) É satisfatório o atendimento psicológico nas clínicas-escolas brasileiras? In R. M. L. L. Carvalho (Ed.), Repensando a formação do psicólogo: Da informação à descoberta: Vol. 1. Coletâneas da ANPEPP (n. 9, pp. 137-145). Campinas, SP: Alínea

Silvares, E. F. M. (2000). Invertendo o caminho tradicional do atendimento psicológico numa clínica-escola brasileira. Estudos de Psicologia (Natal), 5(1), 149-180.

Terzis, A., \& Carvalho, R. M. L. L. (1986). Certas características da população atendida na Clínica de Pós-Graduação PUCCAMP. Estudos de Psicologia (Campinas), 1/2, 112-128.

Terzis, A., \& Carvalho, R. M. L. L. (1988). Identificação da população atendida na clínica-escola do Instituto de Psicologia da PUCCAMP. Arquivos Brasileiros de Psicologia, 40(4), 87-97.

Wolf, S. M. R. (1988). Alguns dados sobre a caracterização da clientela do Centro de Psicologia Aplicada da UNESP. Perfil: Boletim de Psicologia, 1(1), 78-96.

Yehia, G. Y. (1994). Caracterização da clientela que procura o serviço de identificação de superdotados numa clínica psicológica. Estudos de Psicologia (Campinas), 11(3), 3-9. 